\title{
39. STABLE ISOTOPE RECORDS IN BULK SEDIMENTS (LEG 138) ${ }^{1}$
}

\author{
N.J. Shackleton ${ }^{2}$ and M.A. Hall ${ }^{2}$
}

\begin{abstract}
Several hundred samples of bulk sediments from all the 11 sites drilled during Ocean Drilling Program (ODP) Leg 138 in the eastern equatorial Pacific Ocean have been analyzed for $\delta^{13} \mathrm{C}$ and $\delta^{18} \mathrm{O}$. The $\delta^{13} \mathrm{C}$ records provide information on the changes in the operation of the global carbon cycle during the late Neogene that were postulated by Shackleton (1985) on the basis of analogous data from Deep Sea Drilling Program (DSDP) Leg 74 in the South Atlantic. The general validity of the trend from isotopically heavier $\delta^{13} \mathrm{C}$ values during the mid-Miocene toward lighter values today is confirmed. The data appear to be useful for high-resolution correlation among sites. The origin of the variability is enigmatic; even in the Pleistocene, there is low-frequency variability in bulk sediment $\delta^{13} \mathrm{C}$ having an amplitude of more than $1 \%$ that is not present in $\delta^{13} \mathrm{C}$ records from foraminifers, and the concerns expressed by Broecker and Woodruff (1990) regarding the interpretation of the record must be taken seriously.

The bulk-sediment $\delta^{18} \mathrm{O}$ data for the past $1 \mathrm{~m}$.y. show a latitudinal trend that is consistent with the present-day surface temperature gradient, with cooler water on the equator caused by upwelling. The data for the interval 1 to $4 \mathrm{Ma}$ show a similar trend, but between 4 and $7 \mathrm{Ma}$, the gradient across latitude is steeper. When interpreted in terms of temperature, this implies that surface temperatures along the equator were lower during the interval of exceptionally high sediment accumulation rates of the latest Miocene and early Pliocene. This intense equatorial upwelling declined at about $4 \mathrm{Ma}$, perhaps as the connection to the Atlantic Ocean across Middle America became ineffective.

In the sediment immediately above 11 - to 12 -Ma-old basement, the $\delta^{18} \mathrm{O}$ values at Sites $849,850,851$, and 852 become isotopically heavier than the values for sediment of similar age from Site 845 (which lies on 16-Ma-old basement and preserves a longer record). This may imply diagenetic exchange at a relatively low temperature (altering the $\delta^{18} \mathrm{O}$ value toward a more positive value). Overall, the data sets are remarkably consistent. This suggests that other than close to basement, the isotopic effects of diagenetic change must be small and spatially smooth. It should be possible to retrieve long sea-surface temperature records of immense value from these data.
\end{abstract}

\section{INTRODUCTION}

In a series of papers (Shackleton, 1985; Shackleton, 1986; Shackleton, 1987; Shackleton and Hall, 1984), we reasoned that to learn more about changes in the global carbon system, it is necessary to obtain a historical record of the carbon isotopic composition of bulk marine carbonates as a step toward obtaining an integrated budget for the stable carbon isotopes. This is important because it provides the means for monitoring changes in the global partitioning of carbon between reduced carbon (organic carbon) and oxidized carbon (inorganic carbon, carbonate carbon) and, potentially, for discovering the history of oxygen in the atmosphere. Broecker and Woodruff (1992) cautioned against relying too heavily on a single data set when making inferences about the global carbon system. We had been aware of this danger and had been gathering additional data sets; however, the Leg 138 cruise provided the first opportunity for obtaining first-rate continuous data for the late Neogene with good age control.

Several workers have made useful paleoclimatic inferences from $\delta^{18} \mathrm{O}$ data in bulk sediments (Margolis et al., 1975; Anderson and Steinmetz, 1981; Shackleton, 1986; Shackleton et al., 1993), and a second objective was to explore the potential of $\delta^{18} \mathrm{O}$ studies in bulk sediments in relation to the Leg 138 sequences. This is of interest because, although some of the sites contain sufficient foraminifers to permit the construction of useful $\delta^{18} \mathrm{O}$ records, the preservation of planktonic species is very poor in some of the sites, especially those that lie farthest from the equator.

We have analyzed sediment from all the sites drilled during Leg 138 and have covered almost all intervals represented by carbonate sediments. Figure 1 shows the present positions of the sites (Table 1) and their backtrack paths in 1-m.y. intervals (Shipboard Scientific Party,

\footnotetext{
1 Pisias, N.G., Mayer, L.A., Janecek, T.R., Palmer-Julson, A., and van Andel, T.H (Eds.), 1995. Proc. ODP, Sci. Results, 138: College Station, TX (Ocean Drilling Program).

${ }^{2}$ University of Cambridge, Godwin Laboratory, Free School Lane, Cambridge CB2 3RS, United Kingdom
}

1992, Fig. 3), superimposed on a sea-surface temperature map for August (Levitus, 1982). Over the western transect, the basement age is latest Middle Miocene at Site 848, and slightly younger at Site 854 at the Northern end. On the eastern transect Site 847 lies on younger crust while Sites 844, 845 and 846 lie on latest Early Miocene crust. The sites were chosen to provide information on the history of the surface ocean circulation in the eastern equatorial Pacific. The western transect is well suited for an examination of changes in North-South gradients associated with the equatorial circulation. The interpretation of changes in East-West gradients is complicated by the effects of plate motion on the relative positions of the sites (Fig. 1).

\section{METHODS AND DATA}

Most of the stable isotope data generated in our laboratory over the past three decades have been obtained using material that was vacuumroasted at $450^{\circ} \mathrm{C}$ for $30 \mathrm{~min}$ to drive off organic matter that might contaminate the carbon dioxide to be analyzed in the mass spectrometer (Shackleton and Opdyke, 1973). This procedure was developed as an alternative to the method devised by Epstein et al. (1953), whereby the samples were roasted in a stream of helium for the same purpose. On two occasions, we experienced analytical problems that were traced to a fault in the temperature controller of the roasting oven. These occurrences led us to use hydrogen peroxide, instead of vacuum-roasting, to clean foraminiferal carbonate. In the present study, we initially used the vacuum-roasting method for bulk sediments, but we experienced serious difficulty with contamination in some intervals. For this reason, all measurements were repeated using hydrogen peroxide, and only those results are reported here. Despite the fact that spot checks suggest that in most samples vacuum-roasting generates equally valid data, we recommend that in future vacuum roasting should be avoided or used with caution.

To perform the hydrogen peroxide cleaning, about $0.1 \mathrm{~g}$ of sediment was placed in a glass thimble, covered with about $0.2 \mathrm{~mL}$ hydrogen peroxide (about $3 \%$ concentration) and left for more than $0.5 \mathrm{hr}$. 
Table 1. Location of sites from which data are reported.

\begin{tabular}{cccc}
\hline Site & $\begin{array}{c}\text { Latitude } \\
\left({ }^{\circ}\right)\end{array}$ & $\begin{array}{c}\text { Longitude } \\
\left({ }^{\circ}\right)\end{array}$ & $\begin{array}{c}\text { Depth } \\
(\mathrm{mbsf})\end{array}$ \\
\hline 844 & $7^{\circ} 55.279^{\prime} \mathrm{N}$ & $90^{\circ} 28.846^{\prime} \mathrm{W}$ & 3414.5 \\
845 & $9^{\circ} 34.950^{\prime} \mathrm{N}$ & $94^{\circ} 35.448^{\prime} \mathrm{W}$ & 3704.2 \\
846 & $3^{\circ} 05.696^{\prime} \mathrm{S}$ & $90^{\circ} 49.078^{\prime} \mathrm{W}$ & 3307.3 \\
847 & $0^{\circ} 11.593^{\prime} \mathrm{N}$ & $95^{\circ} 19.227^{\prime} \mathrm{W}$ & 3334.3 \\
848 & $2^{\circ} 59.634^{\prime} \mathrm{S}$ & $110^{\circ} 28.791^{\prime} \mathrm{W}$ & 3853.4 \\
849 & $0^{\circ} 10.983^{\prime} \mathrm{N}$ & $110^{\circ} 31.183^{\prime} \mathrm{W}$ & 3837.1 \\
850 & $1^{\circ} 17.837^{\prime} \mathrm{N}$ & $110^{\circ} 31.283 \mathrm{~W}$ & 3786.1 \\
851 & $2^{\circ} 46.223^{\prime} \mathrm{N}$ & $110^{\circ} 34.308^{\prime} \mathrm{W}$ & 3761.3 \\
852 & $5^{\circ} 17.566^{\prime} \mathrm{N}$ & $110^{\circ} 04.579^{\prime} \mathrm{W}$ & 3861.0 \\
853 & $7^{\circ} 12.661^{\prime} \mathrm{N}$ & $109^{\circ} 45.084^{\prime} \mathrm{W}$ & 3726.0 \\
854 & $11^{\circ} 13.433^{\prime} \mathrm{N}$ & $109^{\circ} 35.652^{\prime} \mathrm{W}$ & 3567.9 \\
\hline
\end{tabular}

It then was rinsed by filling the thimble with pure acetone; the acetone was decanted onto a paper tissue, and the sediment was dried overnight in an oven at $60^{\circ} \mathrm{C}$. About $30 \mathrm{mg}$ of sediment was used for each mass spectrometric analysis to provide ample carbon dioxide for rapid isotopic analysis. The samples were processed in a VG Isotech Autocarb online system and analyzed using a VG SIRA Series II mass spectrometer. The laboratory standard Carrara marble was calibrated to PDB through NBS 19, using the values $-2.20 \%$ for $\delta^{18} \mathrm{O}$ and $+1.95 \%$ for $\delta^{13} \mathrm{C}$ recommended by Coplen (1988). Analytical reproducibility as indicated by the marble analyses is typically about $\pm 0.06 \%$ o $(1-\sigma)$ for $\delta^{18} \mathrm{O}$ and somewhat better for $\delta^{13} \mathrm{C}$

We have also compared measurements performed with untreated sediments and sediments treated with hydrogen peroxide. We were motivated by Schrag (pers. comm., 1992), who suggested that isotopic exchange might occur between the carbonate and any $\mathrm{CO}^{2}$ that had been released by the reaction of hydrogen peroxide with organic matter. Initial results indicate that no systematic difference exists between the two methods for these samples.

The majority of the samples reported here for Leg 138 sites were splits of scrape samples (Leinen et al., this volume). Each scrape sample comprises material removed from the surface of one section (normally $1.5 \mathrm{~m}$ in length, less for the last section in each core) of the core, in preparation for color scanning. Samples were freeze-dried, homogenized, and split in Leinen's laboratory. Aliquots were sent to Cambridge for isotope analysis. In addition, some discrete samples were analyzed; these include samples scraped from U-channels from Hole $848 \mathrm{C}$ and discrete samples taken for magnetic analysis from Hole 852D.

All measurements are listed in Table 2 (CD-Rom, back pocket) with reference to the PDB standard using the standard $\delta$ notation where $\delta^{18} \mathrm{O}=1000\left(\mathrm{R}_{s d} / \mathrm{R}_{s t}-1\right)$ with $R_{s a}$ being the ${ }^{18} \mathrm{O} /{ }^{16} \mathrm{O}$ in the sample gas and $R_{s t}$ the ${ }^{18} \mathrm{O} /{ }^{16} \mathrm{O}$ ratio in the standard. The age models given by Shackleton et al. (this volume) have been used to express all data on a common age scale. The age is estimated for the mid-point of the interval represented by the scrape samples.

\section{DISCUSSION: CARBON ISOTOPES}

Figure 2 shows the carbon isotope data for all sites plotted vs. age. It is apparent that to a close approximation all sites display the same pattern, with a transition from values of above $+2.5 \%$ for the middle Miocene to values close to zero on the PDB scale in late Pleistocene sediments. These values are similar to those obtained from DSDP Hole 525A (Shackleton and Hall, 1984) and to unpublished data from the low-latitude Atlantic and Indian oceans.

When using these data, it is important to appreciate the basic difference between the history of $\delta^{13} \mathrm{C}$ of ocean dissolved inorganic carbon, and the history of $\delta^{13} \mathrm{C}$ in bulk carbonates. As we argued earlier, it is essential to obtain a history of $\delta^{13} \mathrm{C}$ in carbonate deposited from the ocean if we are to unravel the history of the global carbon budget and perhaps of atmospheric oxygen (Shackleton, 1985; Kump and Garrels, 1986). Broecker and Woodruff (1992) explicitly stated that the history of ocean surface water $\delta^{13} \mathrm{C}$, which is monitored (in an ideal world) by measuring $\delta^{13} \mathrm{C}$ in fossil planktonic foraminiferal carbonate, is equiva-

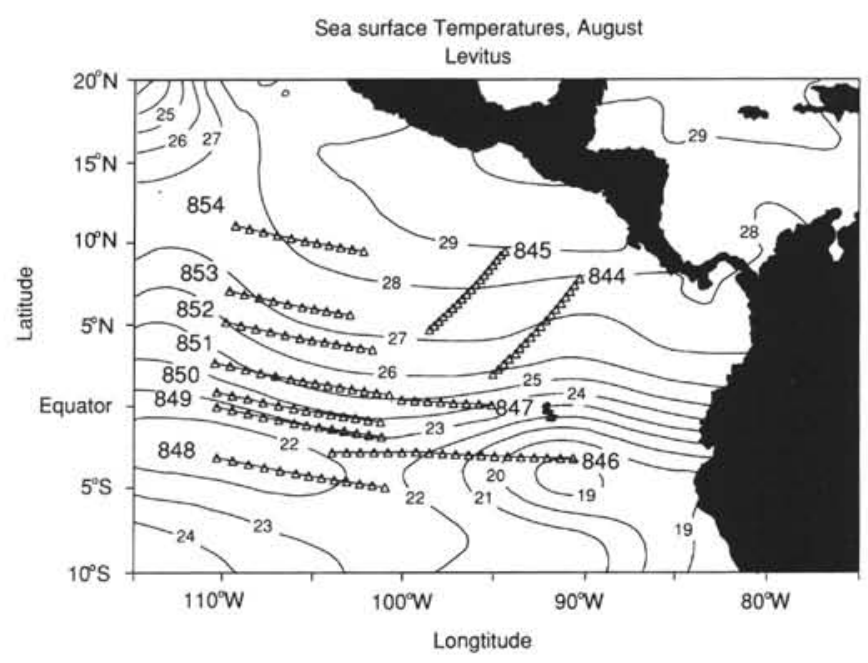

Figure 1. Location of Leg 138 sites and their backtracked paths at 1-m.y. intervals (Mayer, Pisias, Janecek, et al., 1992), superimposed on sea-surface temperatures for August (Levitus, 1982).

lent to the history of $\delta^{13} \mathrm{C}$ in accumulated sediments. They then compared their $\delta^{13} \mathrm{C}$ record derived from analyses of planktonic foraminifera with the record published by Shackleton and Hall (1984) and found that the two records are different; they concluded that this difference constitutes a problem. However, to assume that bulk sediments and surface water have had the same history for $\delta^{13} \mathrm{C}$ requires the hypothesis that the $\delta^{13} \mathrm{C}$ fractionation between surface water and average calcifying organisms has remained constant. Given the rapid evolutionary changes that occurred within the coccoliths, and the good evidence that they do not calcify in isotopic equilibrium for ${ }^{13} \mathrm{C}$ (Dudley and Goodney, 1979), this is not a particularly plausible hypothesis. We would argue that changes in the ${ }^{13} \mathrm{C}$ isotopic difference between $\mathrm{Glo}$ bigerinoides sacculifer and bulk sediments, discussed by Broecker and Woodruff (1992), more likely reflect changes in the average fractionation characterizing the mix of organisms making up the bulk, rather than reflecting changes in the fractionation by $G$. sacculifer as hypothesized by Broecker and Woodruff (1992). Although changes in this difference between bulk sediment and surface water $\delta^{13} \mathrm{C}$ may not be inherently interesting, provided that one does not misuse one record as a proxy for the other, there may even so be cause for concern. The reason is that Shackleton (1985) explicitly assumed that the $\delta^{13} \mathrm{C}$ record of bulk deep-sea sediment is the best approximation to the $\delta^{13} \mathrm{C}$ record of the total carbonate accumulating. However, if the record of ocean surface water $\delta^{13} \mathrm{C}$ is significantly different from the record preserved in bulk deep-sea sediment, then there is no reason to assume that the $\delta^{13} \mathrm{C}$ history of other important carbonate reservoirs (especially shelf carbonate) exactly parallels that of deep-sea sediment.

Figure 2 shows no evidence for a significant long-term change in the $\delta^{13} \mathrm{C}$ of bulk sediments at the time of $\mathrm{C} 3 \mathrm{Ar}$ (Epoch 6 in old terminology). The data gathered by the CENOP group and summarized by Haq et al. (1980) points to a change in $\delta^{13} \mathrm{C}$ in benthic foraminifers that averaged about $0.7 \%$ over an interval of about $0.2 \mathrm{Ma}$ in the middle of Epoch 6 in their terminology. On the time scale of this study (Shackleton et al., this volume), this would be between about 7 to 6.8 Ma. Broecker and Woodruff (1992) considered that because they did not observe such a change in the data shown by Shackleton and Hall (1984), there might have been a defect in these authors' time scale. In fact, there is even less reason to expect a parallelism between $\delta^{13} \mathrm{C}$ in bulk sediments and in the $\delta^{13} \mathrm{C}$ of ocean deep water that records the so-called Epoch 6 carbon shift, than to expect a parallelism between $\delta^{13} \mathrm{C}$ in bulk sediment and in surface water. This is because the $\delta^{13} \mathrm{C}$ of ocean deep water differs from that of surface water by an amount that is also variable on geological time scales (Broecker, 1982); since the isotopic balance at steady state is maintained through $\delta^{13} \mathrm{C}$ in the 
Table $2 .{ }^{13} \mathrm{C}$ and ${ }^{18} \mathrm{O}$ data for Leg 138 sites.

\begin{tabular}{|c|c|c|c|c|c|c|c|c|}
\hline Sample & $\begin{array}{l}\text { Depth } \\
\text { (mbsf) }\end{array}$ & $\begin{array}{c}\text { Depth } \\
\Delta \mathrm{m}\end{array}$ & $\begin{array}{l}\text { Depth } \\
\text { (mcd) }\end{array}$ & $\begin{array}{l}\text { Age } \\
\text { (Ma) }\end{array}$ & $\begin{array}{l}\text { Laboratory } \\
\text { code }\end{array}$ & $\delta^{18} \mathrm{O}$ & $\delta^{13} \mathrm{C}$ & Sample \\
\hline 138-844B- & & & & & & & & \\
\hline IH-1,0-150 & 0.75 & 0.0 & 0.75 & 0.0955 & S93/1059 & 0.58 & 0.19 & $15 \mathrm{H}-4,0-150$ \\
\hline IH-3,0-150 & 3.75 & 0.0 & 3.75 & 0.2929 & S93/1060 & 0.23 & 0.68 & $15 \mathrm{H}-5,0-150$ \\
\hline $\begin{array}{l}2 \mathrm{H}-1,0-150 \\
2 \mathrm{H}-2,0-150\end{array}$ & 5.25 & 1.1 & 6.38 & $\begin{array}{l}0.4759 \\
0.5852\end{array}$ & S93/1061 & 0.37 & 1.29 & $15 \mathrm{H}-6,0-150$ \\
\hline $\begin{array}{l}2 \mathrm{H}-2.0-150 \\
2 \mathrm{H}-4,0-50\end{array}$ & $\begin{array}{l}6.75 \\
975\end{array}$ & 1.1 & $\begin{array}{r}7.88 \\
1088\end{array}$ & $\begin{array}{l}0.5853 \\
0.8770\end{array}$ & $\begin{array}{l}S 93 / 1062 \\
S 93 / 1063\end{array}$ & -0.03 & 0.19 & $15 \mathrm{H}-7,0-150$ \\
\hline $2 \mathrm{H}-5,0-150$ & 11.25 & 1.1 & 12.38 & $\begin{array}{l}0.8770 \\
1.0450\end{array}$ & $\begin{array}{l}\text { S93/1063 } \\
\text { S93/1064 }\end{array}$ & $\begin{array}{l}0.39 \\
0.08\end{array}$ & $\begin{array}{l}0.55 \\
0.61\end{array}$ & $\begin{array}{l}16 \mathrm{H}-1,0-150 \\
16 \mathrm{-}-0-150\end{array}$ \\
\hline $2 \mathrm{H}-6,0-150$ & 12.75 & 1.1 & 13.88 & 1.2300 & S93/1065 & $\begin{array}{l}0.00 \\
-0.09\end{array}$ & 0.48 & $16 \mathrm{H}-3,0-150$ \\
\hline $2 \mathrm{H}-7,0-150$ & 13.99 & 1.1 & 15.12 & 1.3886 & S93/1066 & -0.24 & 0.79 & $16 \mathrm{H}-4,0-150$ \\
\hline $3 \mathrm{H}-1,0-150$ & 14.75 & 2.2 & 17.00 & 1.6292 & $593 / 1067$ & -0.03 & -0.21 & $16 \mathrm{H}-5,0-150$ \\
\hline $3 \mathrm{H}-2,0-150$ & 16.25 & 2.2 & 18.50 & 1.8386 & S93/1068 & -0.18 & 0.64 & $16 \mathrm{H}-6,0-150$ \\
\hline $3 \mathrm{H}-3,0-150$ & 17.75 & 2.2 & 20.00 & 2.1311 & S93/1069 & -0.25 & 0.17 & $16 \mathrm{H}-7,0-150$ \\
\hline $3 \mathrm{H}-4.0-150$ & 19.25 & 2.2 & 21.50 & 2.4508 & S93/1070 & -0.32 & 0.29 & $17 \mathrm{H}-1,0-150$ \\
\hline $3 \mathrm{H}-5,0-150$ & 20.75 & 2.2 & 23.00 & 2.8338 & $S 93 / 1071$ & -0.05 & 0.01 & $17 \mathrm{H}-2,0-150$ \\
\hline $3 \mathrm{H}-6,0-150$ & 22.25 & 2.2 & 24.50 & $\begin{array}{l}3.3370 \\
3.760\end{array}$ & $\$ 93 / 1072$ & -0.25 & 0.08 & i \\
\hline $3 \mathrm{H}-7,0-150$ & 23.53 & 2.2 & 25.78 & $\begin{array}{l}3.7369 \\
14620\end{array}$ & $\begin{array}{l}S 93 / 1073 \\
S 02\end{array}$ & -0.22 & 0.08 & $17 \mathrm{H}-4,0-150$ \\
\hline $4 \mathrm{H}-1,0-150$ & $\begin{array}{l}24.25 \\
75.75\end{array}$ & -8.5 & 15.70 & $\begin{array}{l}1.4629 \\
16548\end{array}$ & $\begin{array}{l}S 93 / 1074 \\
\$ 93 / 1075\end{array}$ & -0.05 & 0.45 & $17 \mathrm{H}-5,0-150$ \\
\hline $4 \mathrm{H}-2,0-150$ & $\begin{array}{l}25.75 \\
27.25\end{array}$ & $\begin{array}{l}-8.5 \\
-8.5\end{array}$ & $\begin{array}{l}17.20 \\
1870\end{array}$ & $\begin{array}{l}1.6548 \\
18729\end{array}$ & $\begin{array}{l}\text { S93/1075 } \\
\text { S93/1076 }\end{array}$ & -0.40 & -0.22 & $17 \mathrm{H}-6,0-150$ \\
\hline $\begin{array}{l}4 \mathrm{H}-3,0-150 \\
4 \mathrm{H}-3,0-150\end{array}$ & $\begin{array}{l}27.25 \\
27.25\end{array}$ & $\begin{array}{l}-8.5 \\
-8.5\end{array}$ & $\begin{array}{l}18.70 \\
18.70\end{array}$ & $\begin{array}{l}1.8729 \\
1.8729\end{array}$ & & $\begin{array}{l}0.20 \\
0.87\end{array}$ & $\begin{array}{r}-1.15 \\
0.70\end{array}$ & $\begin{array}{l}17 \mathrm{H}-7.0-150 \\
18 \mathrm{H}-1,0-150\end{array}$ \\
\hline $4 \mathrm{H}-4,0-150$ & 28.75 & $\begin{array}{l}-6.03 \\
-8.5\end{array}$ & 20.20 & 2.1738 & S93/1077 & $\begin{array}{r}0.81 \\
-0.44\end{array}$ & -0.10 & $18 \mathrm{H}-2,0-150$ \\
\hline $4 \mathrm{H}-5,0-150$ & 30.25 & -8.5 & 21.70 & 2.4934 & S93/1078 & -0.33 & 0.30 & $18 \mathrm{H}-3,0-150$ \\
\hline $4 \mathrm{H}-6.0-150$ & 31.75 & -8.5 & 23.20 & 2.8923 & S93/1079 & -0.09 & 0.03 & $18 \mathrm{H}-4,0-150$ \\
\hline $0-150$ & 32.99 & -8.5 & 24.44 & 3.2692 & $\$ 93 / 1080$ & -0.29 & 0.28 & $18 \mathrm{H}-5,0-150$ \\
\hline $5 \mathrm{H}-1,0-150$ & 33.75 & 3.6 & 37.40 & 6.1957 & S93/1081 & -0.99 & 0.29 & $18 \mathrm{H}-6,0-150$ \\
\hline $5 \mathrm{H}-2,0-150$ & 35.25 & 3.6 & 38.90 & 6.5857 & S93/1082 & -1.03 & 0.21 & $18 \mathrm{H}-7,0-150$ \\
\hline $5 \mathrm{H}-3,0-150$ & 36.75 & 3.6 & 40.40 & 6.8238 & $593 / 1083$ & -0.91 & 0.54 & $19 \mathrm{H}-1,0-150$ \\
\hline $5 \mathrm{H}-4,0-150$ & 38.25 & 3.6 & 41.90 & 7.0967 & $593 / 1$ & -1.01 & 0.68 & $19 \mathrm{H}-2,0-150$ \\
\hline $5 \mathrm{H}-5,0-150$ & 39.75 & 3.6 & 43.40 & 7.4314 & S93/ & -1.20 & 0.64 & $19 \mathrm{H}-3,0-150$ \\
\hline $5 \mathrm{H}-6,0-150$ & 41.25 & 3.6 & 44.90 & 7.6103 & S93/1086 & -0.99 & $\begin{array}{l}1.38 \\
0.88\end{array}$ & $19 \mathrm{H}-4,0-150$ \\
\hline $5 \mathrm{H}-7,0-150$ & 42.45 & 3.6 & 46.10 & $\begin{array}{l}7.7121 \\
7.8143\end{array}$ & $\begin{array}{l}\$ 93 / 1087 \\
\$ 93 / 088\end{array}$ & -1.13 & 0.88 & $19 \mathrm{H}-5,0-150$ \\
\hline $6 \mathrm{H}-1,0-1500$ & $\begin{array}{l}43.25 \\
44.75\end{array}$ & 4.1 & $\begin{array}{l}47.35 \\
48.85\end{array}$ & $\begin{array}{l}7.8143 \\
7.9370\end{array}$ & $\begin{array}{l}\text { S93/I088 } \\
\text { S93/1089 }\end{array}$ & -1.09 & $\begin{array}{l}1.01 \\
0.98\end{array}$ & $19 \mathrm{H}-6,0-150$ \\
\hline $6 \mathrm{H}-3,0-150$ & 46.25 & $\begin{array}{l}4.1 \\
4.1\end{array}$ & $\begin{array}{l}48.83 \\
50.35\end{array}$ & 8.0911 & $\begin{array}{l}593 / 1089 \\
\$ 93 / 1090\end{array}$ & $\begin{array}{l}-1.23 \\
-1.08\end{array}$ & 0.07 & $20 \mathrm{H}-\mathrm{I}, 0-150$ \\
\hline $6 \mathrm{H}-4,0-150$ & 47.75 & 4.1 & 51.85 & 8.3314 & S93/1091 & -1.06 & 1.04 & $20 \mathrm{H}-2,0-150$ \\
\hline $6 \mathrm{H}-5,0-150$ & 49.25 & 4.1 & 53.35 & 8.5717 & S93/1092 & -0.89 & 1.05 & $20 \mathrm{H}-3,0-150$ \\
\hline $6 \mathrm{H}-6,0-150$ & 50.75 & 4.1 & 54.85 & 8.7481 & $\$ 93 / 1093$ & -1.03 & 0.94 & $20 \mathrm{H}-4,0-150$ \\
\hline $6 \mathrm{H}-7,0-150$ & 52.01 & 4.1 & 56.11 & 8.8787 & S93/1094 & -0.38 & 0.19 & $20 \mathrm{H}-5,0-150$ \\
\hline $7 \mathrm{H}-1,0-150$ & 52.75 & 6,7 & 59.45 & 9.3034 & S93/1095 & -0.64 & 0.59 & $20 \mathrm{H}-6,0-150$ \\
\hline $7 \mathrm{H}-2,0-150$ & 54.25 & 6.7 & 60.95 & 9.4978 & S93/1096 & -0.06 & 0.18 & $20 \mathrm{H}-7,0-150$ \\
\hline $74-3,0-150$ & 55.75 & 6.7 & 62.45 & 9.6430 & S93/1097 & -0.64 & 0.48 & $21 X-1,0-150$ \\
\hline $7 \mathrm{H}-4,0-150$ & 57.25 & 6.7 & 63.95 & 9.7645 & S93/1221 & -0.76 & 0.83 & $21 \times-2,0-150$ \\
\hline $7 \mathrm{HH}-5,0-150$ & 58.75 & 6.7 & 65.45 & 9.8860 & S93/1099 & -0.88 & 1.21 & $0-150$ \\
\hline $7 \mathrm{H}-6,0-150$ & 60.25 & 6.7 & 66.95 & 10.0074 & S93/1100 & -0.87 & 1.39 & $0-150$ \\
\hline $7 \mathrm{H}-7,0-150$ & 61.53 & 6.7 & 68.23 & 10.1204 & S93/1101 & -0.87 & 1.35 & $0-150$ \\
\hline $8 \mathrm{H}-1.0-150$ & 62.25 & 6.7 & 68.95 & 10.1848 & $S 93 / 1102$ & -0.76 & 1.40 & $21 X-6,0-150$ \\
\hline $\begin{array}{l}8 \mathrm{H}-2,0-150 \\
8 \mathrm{H}-3,0-150\end{array}$ & $\begin{array}{l}63.75 \\
65.25\end{array}$ & 7.1 & $\begin{array}{l}70.90 \\
7240\end{array}$ & $\begin{array}{l}10.3592 \\
10.4934\end{array}$ & $\begin{array}{l}593 / 1103 \\
S 93 / 104\end{array}$ & -0.82 & 1.30 & $21 \times-7,0-150$ \\
\hline $8 \mathrm{H}-4,0-150$ & $\begin{array}{l}05.25 \\
66.75\end{array}$ & 7.1 & $\begin{array}{l}72.40 \\
73.90\end{array}$ & $\begin{array}{l}10.4934 \\
10.6051\end{array}$ & $593 / 1105$ & ${ }_{-0.78}^{-0.72}$ & $\begin{array}{l}1.51 \\
1.57\end{array}$ & $\begin{array}{l}22 X-1,0-150 \\
22 X-2,0-150\end{array}$ \\
\hline $8 \mathrm{H}-5,0-150$ & 68.25 & 7.1 & 75.40 & 10.6990 & $\mathrm{~S} 93 / 1106$ & -0.53 & 1.70 & 3. $0-150$ \\
\hline $8 \mathrm{H}-6,0-150$ & 69.75 & 7.1 & 76.90 & 10.7681 & S93/1107 & -0.65 & 1.57 & $22 \times-4,0-150$ \\
\hline $8 \mathrm{H}-7,0-150$ & 70.98 & 7.1 & 78.13 & 10.8247 & S93/1108 & -1.01 & 1.51 & $22 \times-5,0-150$ \\
\hline $9 \mathrm{H}-1,0-150$ & 71.75 & 7.1 & 78.90 & 10.8602 & $\begin{array}{l}593 / 1109 \\
\end{array}$ & -0.87 & 1.31 & $22 \times-6,0-150$ \\
\hline $9 \mathrm{H}-2,0-150$ & 73.25 & 8.6 & 81.90 & 10.9961 & $\mathrm{~S} 93 / 1222$ & -0.53 & 1.42 & $22 \times-7,0-150$ \\
\hline $9 \mathrm{H}-3,0-150$ & 74.75 & 8.6 & 83.40 & 11.0442 & $S 93 / 1112$ & -0.44 & 1.39 & $23 \times-1,0-150$ \\
\hline $9 \mathrm{H}-4,0-150$ & 76.25 & 8.6 & 84.90 & i1. .0924 & S93/1il3 & -0.17 & 1.71 & $23 \times-2,0-150$ \\
\hline $9 \mathrm{H}-5,0-150$ & 77.75 & 8.6 & 86.40 & 11.1405 & $593 / 1114$ & -0.01 & 2.18 & $23 \times-3,0-150$ \\
\hline $9 \mathrm{H}-6,0-150$ & 79.25 & 8.6 & 87.90 & 11.1886 & S93/1115 & 0.02 & 2. & $23 \times-4,0-150$ \\
\hline $9 \mathrm{H}-7,0-150$ & 80.55 & 8.6 & $\begin{array}{l}89.20 \\
9.88\end{array}$ & 11.2303 & $\begin{array}{l}S 93 / 1116 \\
\$ 93 / 17\end{array}$ & 0.17 & 2.47 & $23 \times-5,0-150$ \\
\hline $10 \mathrm{H}-1,0-150$ & $\begin{array}{l}81.25 \\
82.75\end{array}$ & 10.6 & $\begin{array}{l}91.88 \\
93.38\end{array}$ & 11.3162 & $\begin{array}{l}\text { S93/1117 } \\
\text { S93/1118 }\end{array}$ & -0.02 & 2.21 & $23 X-6.0-150$ \\
\hline $10 \mathrm{H}-3,0-150$ & 84.25 & 10.6 & 94.88 & 11.4057 & $\begin{array}{l}593 / 1119 \\
593118\end{array}$ & 0. & 2. & $\begin{array}{l}23 X-7,0-150 \\
24 \mathrm{X}-1,0-150\end{array}$ \\
\hline $10 \mathrm{H}-4,0-150$ & 85.75 & 10.6 & 9 & 11.4456 & S $93 / 1120$ & 0.05 & 2. & $2,0-150$ \\
\hline $10 \mathrm{H}-5,0-150$ & 87.25 & 10.6 & & 11.4855 & $593 / 1121$ & 0.14 & 2. & 3. $0-150$ \\
\hline $10 \mathrm{H}-6,0-150$ & 88.75 & 10.6 & 99.38 & 11.5254 & S93/1122 & 0.03 & 2. & $4,0-150$ \\
\hline $10 \mathrm{H}-7,0-150$ & 89.89 & 10.6 & 100.52 & 11.5556 & $S 93 / 1123$ & 0.12 & 2.0 & $24 X-5,0-150$ \\
\hline $1 \mathrm{IH}-1,0-150$ & 90.75 & 13.5 & 104.25 & 11.6550 & $S 93 / 1124$ & -0.05 & 1.7 & $24 X-6,0-150$ \\
\hline IIH-2, 0-150 & & 13.5 & 105.75 & 11.6 & S93/1125 & -0.06 & 1.6 & $24 \times-7,0-150$ \\
\hline IiH-3,0-150 & 93.75 & 13.5 & 107.25 & 11.7 & $593 / 1126$ & 0.09 & 1.2 & $25 \times-1,0-150$ \\
\hline & $\begin{array}{l}95.25 \\
96.75\end{array}$ & $\begin{array}{l}13.5 \\
13.5\end{array}$ & $\begin{array}{l}108.75 \\
110.25\end{array}$ & $\begin{array}{l}11.7747 \\
11.8146\end{array}$ & $\begin{array}{l}593 / 1127 \\
593 / 1128\end{array}$ & 0.10 & $\begin{array}{l}1.54 \\
1.33\end{array}$ & $\begin{array}{l}25 \mathrm{X}-2,0-150 \\
25 \mathrm{X}-30-150\end{array}$ \\
\hline $\begin{array}{l}1 \mathrm{H}-5,0-150 \\
\text { IH } 6,0-150\end{array}$ & 98.25 & 13.5 & $\begin{array}{l}110.25 \\
111.75\end{array}$ & $\begin{array}{l}11.8146 \\
11.8545\end{array}$ & $\begin{array}{l}S 93 / 1128 \\
\text { S93/1129 }\end{array}$ & $\begin{array}{l}0.04 \\
0.07\end{array}$ & $\begin{array}{l}1.33 \\
1.50\end{array}$ & $\begin{array}{l}25 X-3,0-150 \\
25 X-0-150\end{array}$ \\
\hline iIH-7,0-150 & 99.50 & 13.5 & 113.00 & 11.88 & S93/1130 & 0.07 & 1.63 & $25 \mathrm{X}-5,0-150$ \\
\hline $12 \mathrm{H}-1,0-150$ & 100.25 & 13 & 113 & 11.9085 & $\begin{array}{l}S 93 / 1131 \\
\end{array}$ & 0.0 & 1.5 & $25 \mathrm{X}-6,0-150$ \\
\hline $12 \mathrm{H}-2,0-1$ & & & & & & 0. & i. & 0 \\
\hline$\Rightarrow$ & & & & & & 0. & i. & $0-1$ \\
\hline$\because$ & & & & & & 0.2 & i. & $n-1$ \\
\hline & & 13 & & & & 0.28 & i. & $0-$ \\
\hline & & & & 12.1512 & & -0.04 & 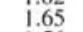 & $0-1$ \\
\hline & & & & & 1137 & & i. & $0-1$ \\
\hline & & 13.8 & 123 & & (11) & -0 & & \\
\hline & & 13.8 & & & & -0 & & 0 i5 \\
\hline & 112. & 13.8 & 126.55 & 12.3 & 59 & 0. & i. & 7. $0-150$ \\
\hline & & $\begin{array}{l}13.8 \\
13.8\end{array}$ & 128 & & & 0.2 & 1.6 & $27 X-C C, 0-150$ \\
\hline 00 & $\begin{array}{l}115.75 \\
117.25\end{array}$ & $\begin{array}{l}13.8 \\
13.8\end{array}$ & $\begin{array}{r}129.55 \\
131.05\end{array}$ & 12.45 & $\begin{array}{l}S 93 / 1142 \\
\$ 93 / 143\end{array}$ & 0.3 & $\begin{array}{l}1.7 \\
1.6\end{array}$ & $\begin{array}{l}27 X-C C, 0-150 \\
28 X-10-150\end{array}$ \\
\hline $\begin{array}{l}3 \mathrm{H}-6,0,0-150 \\
13 \mathrm{H}-7,0-150\end{array}$ & 118.48 & 13.8 & $\begin{array}{l}131.05 \\
132.28\end{array}$ & 12.5490 & $\begin{array}{l}S 93 / 1143 \\
S 934\end{array}$ & 0.33 & 1.6 & $28 \mathrm{X}-1,0-150$ \\
\hline $14 \mathrm{H}-1,0-150$ & & & 134.08 & 12.6143 & $593 / 1145$ & 0.30 & i. & $2,0-150$ \\
\hline & & & & & & 0.2 & $i$ i. & $28 \mathrm{X}-2,0-150$ \\
\hline & & & & & & & & $28 \times-3,0-150$ \\
\hline & & & & & & & & $3,0-150$ \\
\hline & & & & & & & & 4,0 \\
\hline & & & & & 0 & & & 10 \\
\hline & & & & & & & & \\
\hline & & & 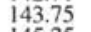 & 0054 & 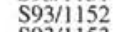 & & & \\
\hline & 0.25 & 15.0 & 145.25 & 12.8678 & $93 / 1153$ & & & $X-7.0-150$ \\
\hline
\end{tabular}

Only the first page of this table is reproduced here. The entire table appears on the CD-ROM (backpocket). 


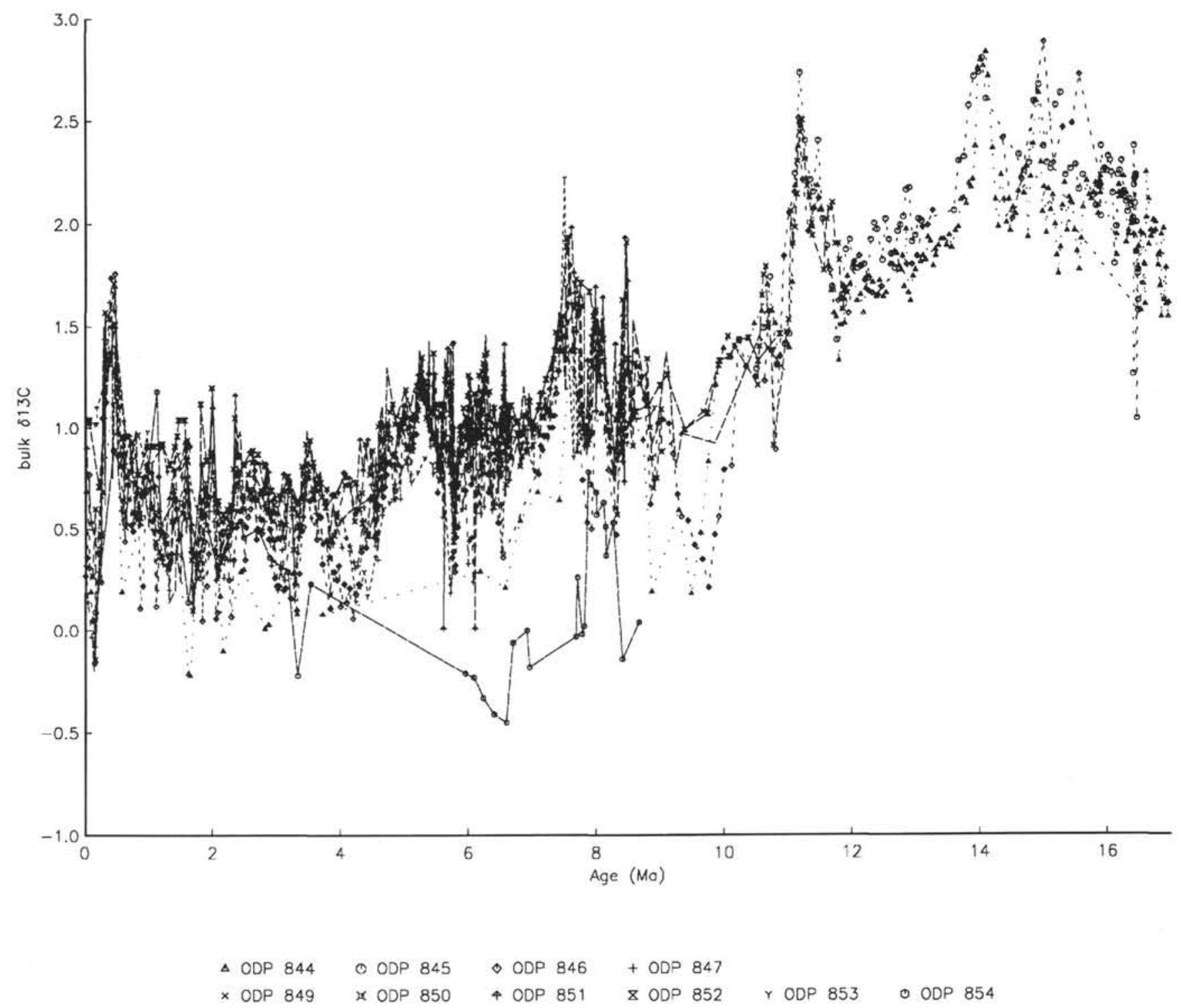

Figure 2. $\delta^{13} \mathrm{C}$ in bulk pelagic sediments vs. age for Leg 138 sites (all data except for Hole 848D).

surface water, this mechanism introduces additional variability in deep water $\delta^{13} \mathrm{C}$.

Table 3 shows mean values in million-year increments. Each value is a simple average of all the measurements falling in the interval; if the sediments of the equatorial Pacific Ocean are representative of global marine carbonates, this table would provide appropriate data for studies of global carbon budgeting. The data in this table differ from those used by Shackleton (1985) in one important respect. The mean value for the last $1 \mathrm{~m} . \mathrm{y}$. is significantly different from the mean value for recent sediments, as given by Shackleton (1985) and by Broecker and Woodruff (1992). As pointed out above, for budgetary purposes, one should use averages taken over about 1 m.y., and it may be that over the last few thousand years, the $\delta^{13} \mathrm{C}$ value of bulk carbonates has been isotopically lighter than the value required for a long-term steady state. This situation may have arisen as a consequence of the dramatic global explosion in the population of Coccolithus huxleyi, which only appeared in the record at about $0.2 \mathrm{Ma}$ and is now the dominant carbonate producer (Thierstein et al., 1977). Shackleton (1985) put forward the argument that when calculating changes in carbon storage and in the concentration of oxygen in the atmosphere, one should start with the values for $\delta^{34} \mathrm{~S}$ and $\delta^{13} \mathrm{C}$ at the present. This is probably incorrect: one should start with a value that has been averaged over a long enough time interval to constitute a steady-state value.

Figure 3 shows the data for the past $12 \mathrm{~m} . \mathrm{y}$. in smaller time segments for clarity. At several points bulk sediment $\delta^{13} \mathrm{C}$ values change by up to $1 \%$ within much less than 1 m.y.; for example, all sites show peak $\delta^{13} \mathrm{C}$ values of about $+1.4 \%$ at $0.4 \mathrm{Ma}$, which may be compared with an average recent value close to zero on the PDB scale (Broecker and Woodruff, 1992) and an average for the last 4 m.y. of $+0.6 \%$ (Table 3). A similar short event is present at about 11.3 Ma. Less marked, but similar, excursions pervade the record. The peak centered at $0.4 \mathrm{Ma}$ was also recorded in the fine fraction from a deep-sea core in the Caribbean analyzed by Anderson and Steinmetz (1981) and in Site 677 by Schrag (pers. comm., 1992). Anderson and Steinmetz (1981) also illustrated the marked changes that have occurred over the past 0.6 M.y. in the makeup of the nannofossil assemblage. Paull and Thierstein (1987) showed that if one separates the fine fraction (less than $3 \mu \mathrm{m}$ ) of a typical deep-sea sediment sample by size class, each size class proves to have been dominated by a different group of nannofossils and has a different $\delta^{13} \mathrm{C}$ (and $\delta^{18} \mathrm{O}$ ) composition; they observed a range of about $3.0 \%$ among size classes from the same sediment sample. Thus, we may anticipate that the evolutionary replacement of one 

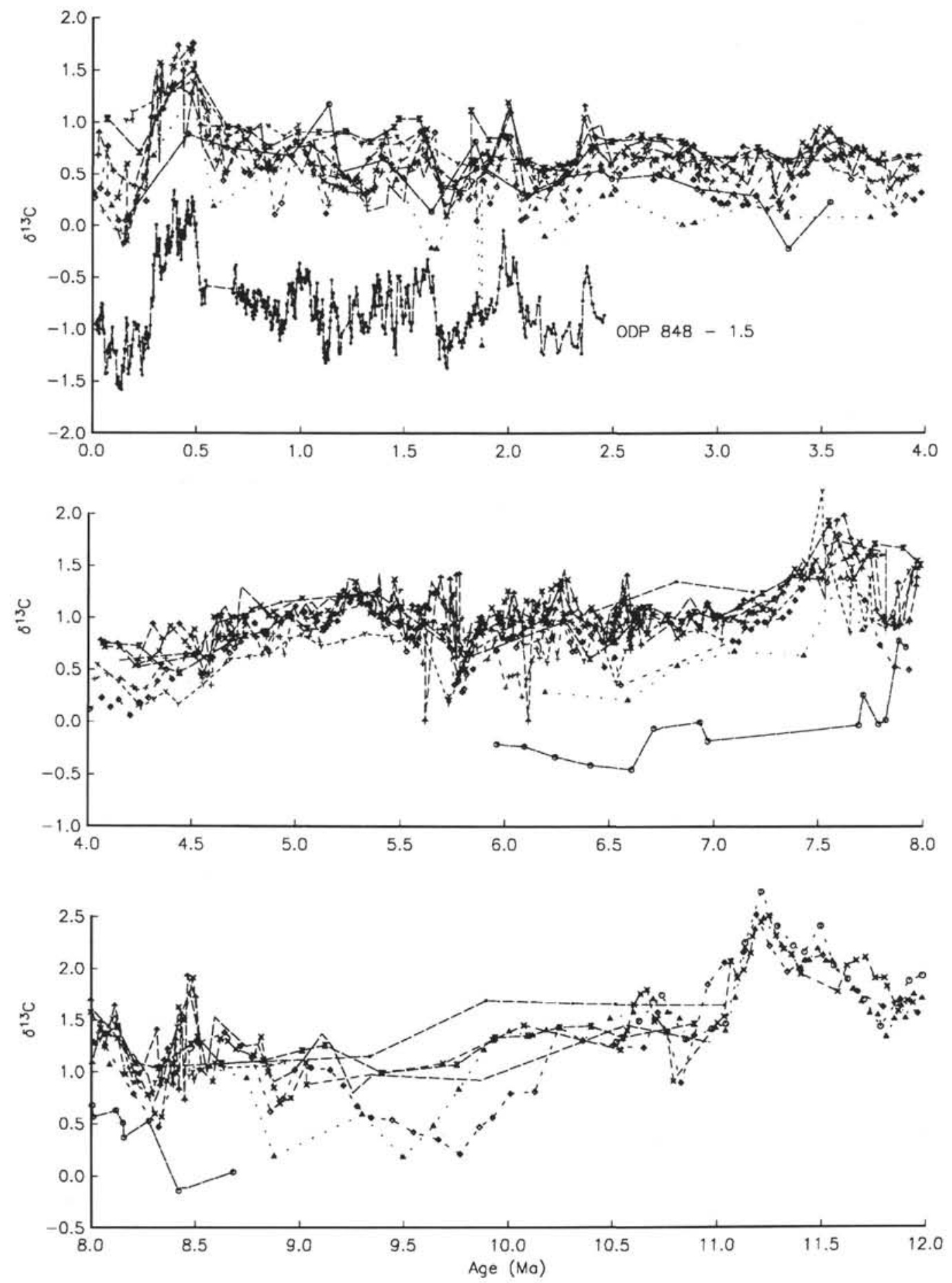
$\triangle$ ODP 844
- ODP 845
- ODP 846
+ ODP 847
- ODP 848
$\times$ ODP $849 \approx$ ODP 850
4 ODP 851
× ODP 852
Y ODP 853
- ODP 854

Figure 3. $\delta^{13} \mathrm{C}$ in bulk pelagic sediments vs. age for Leg 138 sites in 4-m.y. panels. In each site, all data points are shown connected by a line. Note the $1.5 \%$ scale offset for Hole $848 \mathrm{D}$ in the 0 - to 4-Ma panel.

group of nannofossils by another might easily entail a change in the carbon isotopic fractionation between surface ocean dissolved $\mathrm{CO}_{2}$ and accumulating fine carbonates by more than $1 \%$. To maintain a long-term isotopic balance between incoming and outgoing carbon, the isotopic composition of the whole ocean dissolved carbon reservoir would change to counterbalance this alteration in the mean isotopic fractionation factor governing the difference between accu- mulating carbonate and the ocean carbon reservoir. The rate of such a response by the $\delta^{13} \mathrm{C}$ of the dissolved carbon reservoir, of course, is limited by the residence time of carbon, for which Broecker (1974) gives a value of $0.3 \mathrm{~m} . \mathrm{y}$. This figure was obtained by dividing the estimated mass of carbon in the ocean, by the sedimentary output (or erosional influx). The residence time was probably greater prior to the Pleistocene, because global accumulation rates were lower (Donnelly, 
Table 3. $\delta^{13} \mathrm{C}$ mean values for all $\delta^{13} \mathrm{C}$ in each million-year interval.

\begin{tabular}{cc}
\hline $\begin{array}{c}\text { Age } \\
\text { interval } \\
(\mathrm{Ma})\end{array}$ & $\begin{array}{c}\delta^{13} \mathrm{C} \\
\text { to } \\
\text { PDB }\end{array}$ \\
\hline $0-1$ & 0.84 \\
$1-2$ & 0.59 \\
$2-3$ & 0.72 \\
$3-4$ & 0.65 \\
$4-5$ & 0.85 \\
$5-6$ & 1.02 \\
$6-7$ & 1.04 \\
$7-8$ & 1.29 \\
$8-9$ & 1.16 \\
$9-10$ & 1.06 \\
$10-11$ & 1.43 \\
$11-12$ & 1.97 \\
$12-13$ & 1.88 \\
$13-14$ & 2.24 \\
$14-15$ & 2.53 \\
$15-16$ & 2.26 \\
$16-16.5$ & 2.03 \\
\hline
\end{tabular}

1982). This suggests that to be meaningful in relation to global carbon budgeting, the bulk sediment $\delta^{13} \mathrm{C}$ record should be smoothed to a resolution of about $1 \mathrm{Ma}$. High-frequency variations may prove to be useful for stratigraphic correlation, but it will be inherently difficult to interpret $\delta^{13} \mathrm{C}$ in terms of carbon budgeting. High-frequency variations may prove to reflect variations in surface water $\delta^{13} \mathrm{C}$; however, this cannot be assumed without verifying it by analyzing foraminiferal $\delta^{13} \mathrm{C}$.

Stratigraphic correlation has been aided by the $\delta^{13} \mathrm{C}$ record in the neighborhood of the Paleocene/Eocene boundary (Shackleton, 1985). Figure 3 suggests that considerable potential also exists for using the Neogene $\delta^{13} \mathrm{C}$ for the purposes of stratigraphic correlation. Figure 3 also suggests that the high-resolution stratigraphic correlations among the Leg 138 sites proposed by Shackleton et al. (this volume) using the GRAPE density records have been successful.

\section{DISCUSSION: OXYGEN ISOTOPES}

Figure 4 shows oxygen isotope data for bulk sediments in all the Leg 138 sites vs. age. This plot shows significant differences from site to site at all ages. However, a common pattern occurs among Sites 849,850 , and 851 that shows somewhat isotopically lighter values between 1 and $4 \mathrm{Ma}$, and a second more prominent peak at about 12 $\mathrm{Ma}$, flanked by isotopically positive values during the last million years and centered at about $6 \mathrm{Ma}$. The mean difference between the values for the past million years and for the interval from 1 to $4 \mathrm{Ma}$ is about $0.5 \%$ (Table 4 ). If we assume that during the last million years the glacial/interglacial range in ocean water $\delta^{18} \mathrm{O}$ has been about $1.1 \%$, a figure of $0.5 \%$ for a time-averaged enrichment would not be unreasonable. Within the rather large uncertainties, this implies that the trend toward more positive $\delta^{18} \mathrm{O}$ values over the past $1 \mathrm{~m}$.y. may be explicable largely in terms of change in the isotopic composition of the ocean (averaged over glacials and interglacials) and that there was no long-term trend in surface temperature with the growth of glaciation through the last $3 \mathrm{~m} . \mathrm{y}$. This is compatible with the small temperature changes on a glacial/interglacial time scale, documented by CLIMAP (1981) for this region. However, further work will be required before data from bulk sediment can be used to resolve the interesting controversy surrounding the issue of low-latitude temperature changes through the Cenozoic (Barron, 1987).

Although the bulk sediment is a mixture of components, its $\delta^{18} \mathrm{O}$ value does appear to vary with temperature sufficiently closely to be useful. Figure 5 shows measurements (Table 5) for four validated core tops: Site $848 \mathrm{D}$, and piston cores V28-238 ( $\left.1^{\circ} 01^{\prime} \mathrm{N}, 160^{\circ} 29^{\prime} \mathrm{E}\right), \mathrm{RC} 10$ $65\left(0^{\circ} 41.4^{\prime} \mathrm{N}, 108^{\circ} 37.2^{\prime} \mathrm{W}\right)$ and V22-108 $\left(43^{\circ} 11^{\prime} \mathrm{S}, 3^{\circ} 15^{\prime} \mathrm{W}\right)$. Surface water $\delta^{18} \mathrm{O}$ is estimated from Fairbanks et al. (1982) for the East Pacific sites and from Craig and Gordon (1965) for the remainder. Isotopic temperature calculated from $\delta^{18} \mathrm{O}$ is compared with observed warm season temperature (Levitus, 1982). Also plotted are temperatures calculated from the isotopically lightest and heaviest values in each Leg 138
Table 4. $\delta^{18} \mathrm{O}$ mean values.

\begin{tabular}{cccr}
\hline Site & $\begin{array}{c}0-1 \mathrm{Ma} \\
(\%)\end{array}$ & $\begin{array}{c}1-4 \mathrm{Ma} \\
(\%)\end{array}$ & $\begin{array}{r}4-7 \mathrm{Ma} \\
(\%)\end{array}$ \\
\hline 844 & 0.19 & 0.3 & -0.08 \\
846 & 0.85 & 0.30 & 0.15 \\
847 & 0.57 & 0.01 & -0.28 \\
848 & 0.48 & 0.08 & \\
849 & 0.35 & -0.22 & 0.44 \\
850 & 0.16 & -0.34 & 0.24 \\
851 & -0.06 & -0.40 & 0.03 \\
852 & -0.28 & -0.79 & -0.75 \\
853 & -0.12 & -0.65 & -1.06 \\
854 & -0.25 & -1.02 & -1.50 \\
\hline
\end{tabular}

Table 5. Temperature, salinity, and isotope data for Figure 5.

\begin{tabular}{ccrrrrrc}
\hline Site & \multicolumn{1}{c}{$S S T_{s}$} & \multicolumn{1}{c}{ Sal. } & \multicolumn{1}{c}{$\delta^{1 \times} \mathrm{O}_{w}$} & $\delta^{1 \times} \mathrm{O}_{h}$ & $\delta^{18} \mathrm{O}_{l}$ & \multicolumn{1}{c}{$T_{h}$} & \multicolumn{1}{c}{$T_{i}$} \\
\hline ODP846 & 19.0 & 34.7 & 0.05 & 0.25 & 1.72 & 16.0 & 9.8 \\
ODP847 & 24.0 & 34.5 & -0.00 & 0.02 & 1.27 & 16.8 & 11.5 \\
ODP848 & 21.8 & 34.9 & 0.10 & -0.64 & 0.73 & $20.2^{*}$ & $14.2^{*}$ \\
ODP849 & 22.5 & 34.9 & 0.10 & -0.34 & 1.09 & 18.9 & 12.7 \\
ODP850 & 23.3 & 34.9 & 0.10 & -0.43 & 0.74 & 19.3 & 14.1 \\
ODP851 & 24.5 & 34.7 & 0.05 & -0.96 & 0.42 & 21.5 & 15.3 \\
ODP852 & 26.0 & 34.5 & -0.00 & -0.69 & 0.81 & 20.0 & 13.4 \\
ODP853 & 26.6 & 34.0 & -0.13 & -1.04 & 0.10 & 21.0 & 15.9 \\
V28-238 & 28.0 & 34.5 & 0.00 & -2.06 & -0.67 & $26.4^{*}$ & $19.9^{*}$ \\
RC10-65 & 23.3 & 34.9 & 0.10 & -1.03 & 0.61 & $22.0^{*}$ & $14.7^{*}$ \\
V22-108 & 10.4 & 34.3 & -0.40 & 1.72 & 2.89 & $8.0^{*}$ & $3.5^{*}$ \\
\hline
\end{tabular}

Notes: $S S T_{s}=$ sea-surface temperature (August except V22-108, February): $\mathrm{Sal} .=$ salinity: $\delta^{18} \mathrm{O}_{w}=$ surf ace water $\delta^{18} \mathrm{O} ; \delta^{18} \mathrm{O}_{h}=$ most negative bulk $\delta^{18} \mathrm{O}$ measurement $0-1 \mathrm{Ma} ; \delta^{18} \mathrm{O} t=$ most positive bulk $\delta^{18} \mathrm{O}$ measurement $0-1 \mathrm{Ma}: T_{h}=$ temperature calculated from $\delta^{18} \mathrm{O}_{h}\left(^{*}=\right.$ Holocene); $T l=$ temperature calculated from $\delta^{18} \mathrm{O} /\left(^{*}=\right.$ last glacial maximum).

site in the past million years, for several Leg 138 sites. Figure 5 suggests that bulk sediment $\delta^{18} \mathrm{O}$ data give a useful measure of spatial surface temperature distribution and that this may prove to be capable of substantial refinement.

Prior to $4 \mathrm{Ma}, \delta^{18} \mathrm{O}$ values for sediments from Sites 849,850 , and 851 are again more positive, while the values for all other sites are more negative (Table 5). The change is most marked at Site $849(0.65 \%$ heavier). If we can trust these values as indicative of temperature gradients, this would imply that while the average temperature at Site $852\left(5^{\circ} \mathrm{N}\right)$ remained more or less constant, the temperature reduction along the equator caused by equatorial upwelling was accentuated by more than $2^{\circ} \mathrm{C}$ during the early Pliocene, compared either with today or with the Pleistocene and late Pliocene. Figure 6 shows a smooth estimate of the surface $\delta^{18} \mathrm{O}$ gradient between the off-equatorial Sites 852 and 853 , and the near-equator Sites 849,850 , and 851 . To generate this plot, we first estimated $\delta^{18} \mathrm{O}$ values for each site at $0.05-\mathrm{m}$.y. intervals using a Gaussian weighting with a total width of $0.3 \mathrm{~m} . \mathrm{y}$. To generate an off-equator record using the best parts of the records of Sites 852 and 853 , we used only the data for Site 852 between 0 and $2.35 \mathrm{Ma}$; an average of the data from both sites between that age and $4.3 \mathrm{Ma}$; and the data from Site 853 from that age to $8 \mathrm{Ma}$. For the equatorial group, we simply averaged the value for each of Sites 849 , 850 and 851 at every 0.05 m.y. point. Figure 6 shows the two smooth $\delta^{18} \mathrm{O}$ records (top) and the difference (middle); an increase of $1 \%$ in the difference would imply about $4^{\circ}$ of cooling of the equatorial surface water. Figure 6 also shows a smoothed average of the sedimentation rates for Sites 849,850 , and 851 on the $110^{\circ} \mathrm{W}$ transect (estimated over overlapping 0.4-m.y. time intervals), based on the age models of Shackleton et al. (this volume). Figure 6 suggests an association between sedimentation rate along the equator, and cooling of the surface water. The major increase in apparent temperature gradient is associated with a dramatic and sustained increase in sedimentation rates at about $7 \mathrm{Ma}$. The reduction in temperature gradient at about $4.5 \mathrm{Ma}$ is again closely associated with a decrease in sedimentation rates at all sites; sedimentation has remained fairly constant at this lower rate up to the present.

This association between sedimentation rate and temperature on the equator suggests that enhanced wind-driven equatorial circulation, 


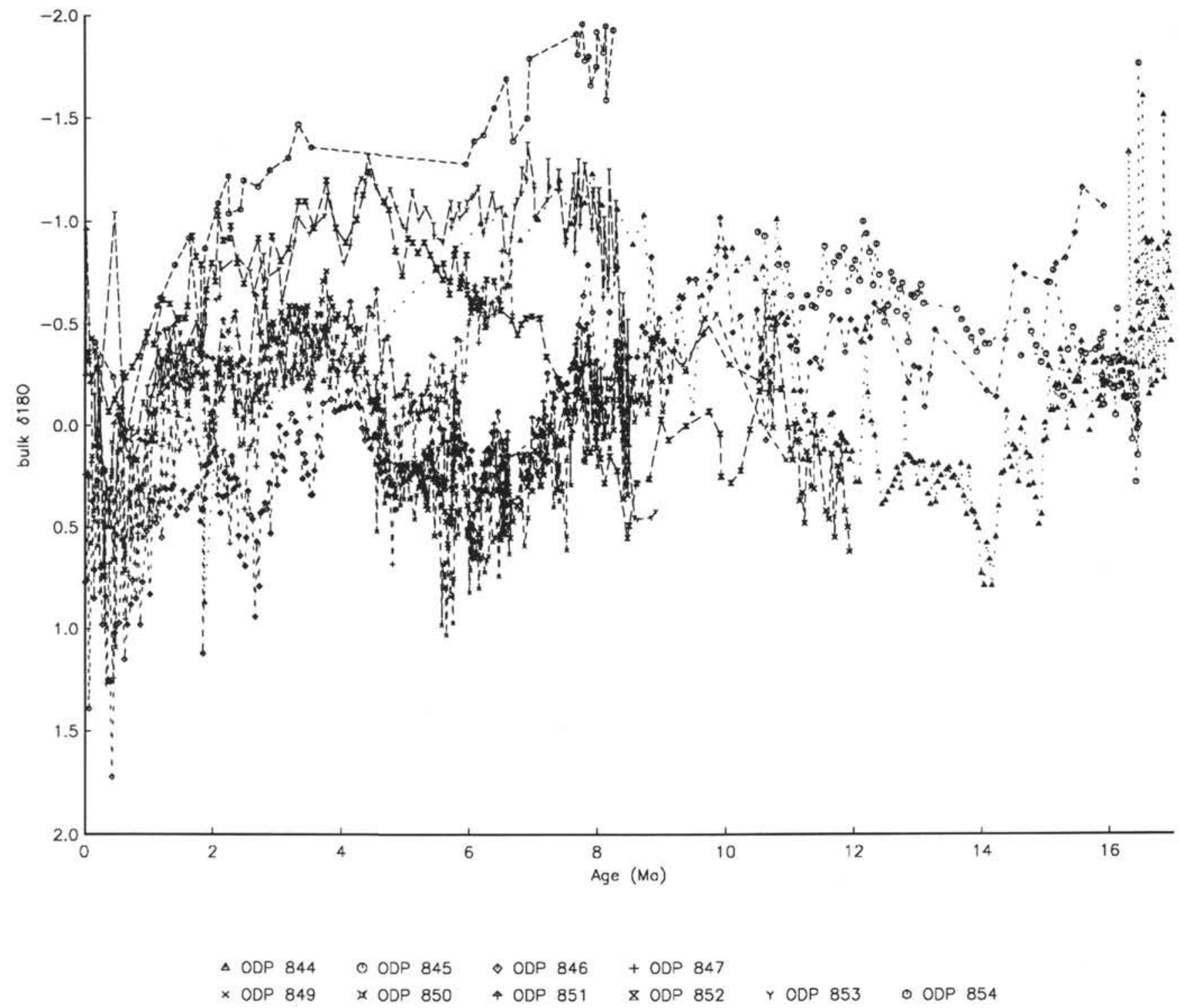

Figure 4. $\delta^{18} \mathrm{O}$ in bulk pelagic sediments vs. age for all Leg 138 sites except Site 848 . The data from the metalliferous sediments at the base of Site 845 are not plotted and would be off the scale.

rather than increased nutrient flux to the oceans, was the cause of the unusually high sedimentation rates in the early Pliocene and late Miocene. Whether the trend toward the lower accumulation rates and reduced temperature gradients of more recent time is linked with the closing of the connection with the Atlantic Ocean across Middle America or whether it is controlled primarily by changes in atmospheric circulation cannot be determined from these data alone. We have not attempted to interpret the data for the period prior to $8 \mathrm{Ma}$ because it is possible that the data from Site 852 has been affected by diagenesis.

In each site, the $\delta^{18} \mathrm{O}$ values take a turn toward more positive values near the base of the section. The data plotted vs. age (Fig. 4) suggest that these trends are not primary, but result from diagenetic alteration of the oldest and most deeply buried sediments. Although the model runs of Schrag et al. (1992) predicted a trend toward isotopically light $\delta^{18} \mathrm{O}$ values in the deepest parts of stratigraphic sections, these workers envisaged interaction with basalt to generate isotopically lighter $\delta^{18} \mathrm{O}$ values in the altered calcite. However, Oyun et al. (this volume) showed that in these sites, active seawater advection occurs in the oceanic crust at the base of the sediments (as first shown for the central equatorial Pacific by Baker et al., 1991), so that the recrystallization trend will be toward low-temperature equilibration with seawater, which is consistent with the observed $\delta^{18} \mathrm{O}$ trends. Only in the metalliferous sediments at the base of the section at Site 845 do we observe very light $\delta^{18} \mathrm{O}$ values that are consistent with equilibration with fluids from the underlying basalt, rather than with seawater.

\section{SUMMARY}

The carbon isotope data from Leg 138 sediments provide a good database with excellent age control for studying global carbon budgeting; to first order, these data confirm the validity of the record from DSDP Leg 74 sediments that has up to now constituted the chief database for this time interval. The carbon isotope records include relatively brief events of enigmatic origin, which may prove valuable for high-resolution stratigraphic correlation. On the other hand, their unexplained existence suggests that the concerns expressed by Broecker and Woodruff (1992) should be taken seriously.

The oxygen isotope data provide a unique record of change in the effect of equatorial upwelling on surface temperature, a record that is consistent with the history of sediment accumulation rates in the region during the Pliocene. However, the use of the oxygen isotope data is probably limited by the effects of diagenesis in the deeper parts 


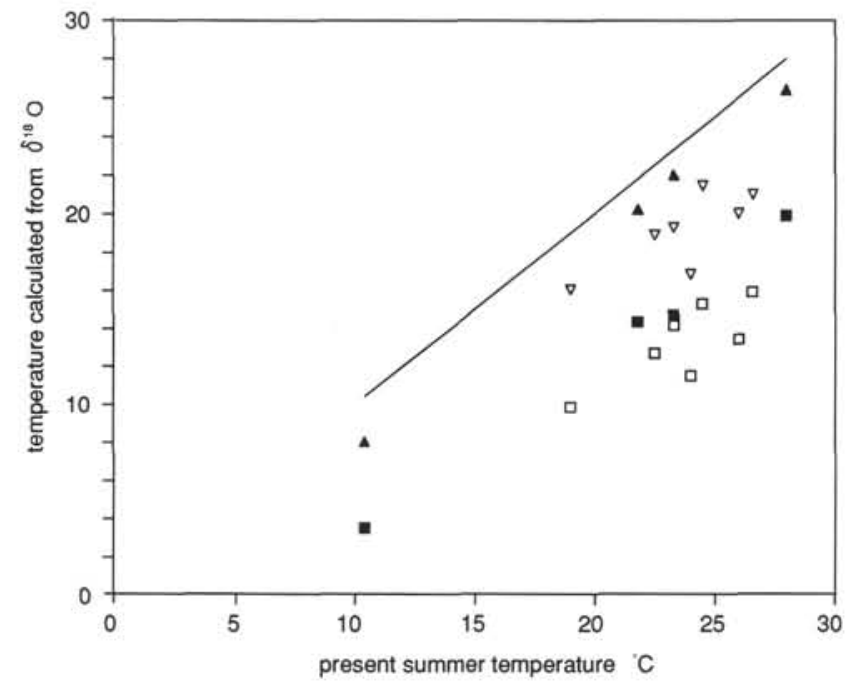

Figure 5. Isotopic temperature calculated from $\delta^{18} \mathrm{O}$ in bulk sediments vs. observed summer sea-surface temperatures (Table 4 ). Solid triangles $=$ validated Holocene data; solid square $=$ validated last glacial maximum; open triangles $=$ warmest scrape sample $0-1 \mathrm{Ma}$; open squares $=$ coolest scrape sample $0-1$ Ma. Temperature is calculated from the relationship $T=16.9-4.4\left(\delta^{18} \mathrm{O}_{c}-\right.$ $\left.\delta^{18} \mathrm{O}_{w}\right)+0.1\left(\delta^{18} \mathrm{O}_{c}-\delta^{18} \mathrm{O}_{w}\right)^{2}$ (O'Neil et al., 1969; Shackleton, 1974).

of the section at all sites; further work should be devoted to this problem, as discussed by Schrag et al. (1992).

\section{ACKNOWLEDGMENTS}

We are deeply indebted to Margaret Leinen, who collected the material scraped from the cores during their preparation aboard ship, and to Charlotte Lehmann, who handled the homogenization and splitting of the samples with meticulous care. This enabled us to take full advantage of the spatial and temporal coverage achieved during Leg 138; for this, in turn, we thank all those aboard who made the cruise so successful. Our analyses in Cambridge were supported by NERC Grant No. GST554, and our preparation of this paper was immensely assisted by Simon Crowhurst. We thank Dan Schrag for sharing and discussing fascinating unpublished data with us, and Mo Raymo, Wally Broecker, Fay Woodruff, and Peggy Delaney for stimulating discussion of the carbon budgeting problems and for helping us to clarify the ideas in our preliminary manuscript.

\section{REFERENCES $*$}

Anderson, T.F., and Steinmetz, J.C., 1981. Isotopic and biostratigraphical records of calcareous nannofossils in a Pleistocene core. Nature, 294:741-744.

Baker, P.A., Stout, P.M., Kastner, M., and Elderfield, H., 1991. Large-scale lateral advection of seawater through oceanic crust in the central equatorial Pacific. Earth Planet. Sci. Lett., 105:522-533.

Barron, E.J., 1987. Eocene equator-to-pole surface ocean temperatures: a significant climate problem? Paleoceanography, 2:729-739.

Broecker, W.S., 1974. Chemical Oceanography: New York (Harcourt Brace Jovanovich).

- 1982. Glacial to interglacial changes in ocean chemistry. Prog. Oceanogr., 11:151-197.

Broecker, W.S., and Woodruff, F., 1992. Discrepancies in the oceanic carbon isotope record for the last fifteen million years? Geochim. Cosmochim. Acta, 56:3259-3264.

\footnotetext{
"Abbreviations for names of organizations and publication titles in ODP reference lists follow the style given in Chemical Abstracts Service Source Index (published by American Chemical Society).
}

CLIMAP Project Members, 1981. Seasonal reconstructions of the Earth's surface at the last glacial maximum. Geol. Soc. Am., Map and Chart Ser, MC36:1-18.

Coplen, T.B., 1988. Normalization of oxygen and hydrogen isotope data. Chem. Geol. (Isotope Geosci. Sect.), 72:293-297.

Craig, H., and Gordon, L.I., 1965. Deuterium and oxygen 18 variations in the ocean and the marine atmosphere. In Tongiorgi, E. (Ed.), Stable Isotopes in Oceanographic Studies and Paleotemperatures, Spoleto: Pisa (Cons. Naz. delle Ric., Lab. di Geol. Nucleare), 9-130.

Donnelly, T.W., 1982. Worldwide continental denudation and climatic deterioration during the late Tertiary: evidence from deep-sea sediments. Geology, $10: 451-454$.

Dudley, W.C., and Goodney, D.E., 1979. Oxygen isotopic content of coccoliths grown in culture. Deep-Sea Res. Part A, 26:495-503.

Epstein, S., Buchsbaum, R., Lowenstam, H.A., and Urey, H.C., 1953. Revised carbonate-water isotopic scale. Geol. Soc. Am. Bull., 64:1315-1325.

Fairbanks, R.G., Sverdlove, M., Free, R., Wiebe, P.H., and Bé, A.W.H., 1982. Vertical distribution and isotopic fractionation of living planktonic foraminifera from the Panama Basin. Nature, 298:841-844.

Haq, B.U., Worsley, T.R., Burckle, L.H., Douglas, R.G., Keigwin, L.D., Jr., Opdyke, N.D., Savin, S.M., Sommer, M.A., II, Vincent, E., and Woodruff, F., 1980. Late Miocene marine carbon-isotopic shift and synchroneity of some phytoplanktonic biostratigraphic events. Geology, 8:427-431.

Kump, L.R., and Garrels, R.M., 1986. Modeling atmospheric $\mathrm{O}_{2}$ in the global sedimentary redox cycle. Am. J. Sci., 286:337-360,

Levitus, S., 1982. Climatological Atlas of the World Ocean. NOAA Prof. Pap., 13.

Margolis, S.V., Kroopnick, P.M., Goodney, D.E., Dudley, W.C., and Mahoney, M.E., 1975. Oxygen and carbon isotopes from calcareous nannofossils as paleoceanographic indicators. Science, 189:555-557.

Mayer, L., Pisias, N., Janecek, T., et al., 1992. Proc. ODP, Init. Repts., 138 (Pts. 1 and 2): College Station, TX (Ocean Drilling Program).

O'Neil, J.R., Clayton, R.N., and Mayeda, T.K., 1969. Oxygen isotope fractionation in divalent metal carbonates. J. Chem. Phys., 51:5547-5558.

Paull, C.K., and Thierstein, H.R., 1987. Stable isotopic fractionation among particles in Quaternary coccolith-sized deep-sea sediments. Paleoceanography, 2:423-429.

Schrag, D.P., DePaolo, D.J., and Richter, F.M., 1992. Oxygen isotope exchange in a two-layer model of oceanic crust. Earth Planet. Sci. Lett., 111:305-317.

Shackleton, N.J., 1974. Attainment of isotopic equilibrium between ocean water and the benthonic foraminifera genus Uvigerina: isotopic changes in the ocean during the last glacial. Les Meth. Quant. d'etude Var. Clim. au Cours du Pleist., Coll. Int. C.N.R.S., 219:203-209.

1985. Oceanic carbon isotope constraints on oxygen and carbon dioxide in the Cenozoic atmosphere. In Sundquist, E.T., and Broecker, W.S. (Eds.), The Carbon Cycle and Atmospheric $\mathrm{CO}_{2}:$ Natural Variations Archean to Present. Am. Geophys Union Monogr., 32:412-417.

, 1986. Paleogene stable isotope events. Palaeogeogr., Palaeoclimat., Palaeoecol., 57:91-102.

, 1987. The carbon isotope record of the Cenozoic: history of organic carbon burial and of oxygen in the ocean and atmosphere. In Brooks, J., and Fleet, A.J. (Eds.), Marine Petroleum Source Rocks. Geol. Soc. Spec. Publ. London, 26:423-434.

Shackleton, N.J., and Hall, M.A., 1984. Carbon isotope data from Leg 74 sediments. In Moore, T.C., Jr., Rabinowitz, P.D., et al., Init. Repts. DSDP, 74: Washington (U.S. Govt. Printing Office), 613-619.

Shackleton, N.J., Hall, M.A., Pate, D., Meynadier, L., and Valet, J.-P., 1993. High resolution stable isotope stratigraphy from bulk sediment. Paleoceanography, 8:141-148.

Shackleton, N.J., and Opdyke, N.D., 1973. Oxygen isotope and paleomagnetic stratigraphy of equatorial Pacific core V28-238: oxygen isotope temperatures and ice volumes on a $10^{5}$ year and $10^{6}$ year scale. Quat. Res., 3:39-55.

Shipboard Scientific Party, 1992. Introduction. In Mayer, L., Pisias, N., Janecek, T., et al., Proc. ODP, Init. Repts., 138 (Pt. 1): College Station, TX (Ocean Drilling Program), 5-12.

Thierstein, H.R., Geitzenauer, K., Molfino, B., and Shackleton, N.J., 1977. Global synchroneity of late Quaternary coccolith datum levels: validation by oxygen isotopes. Geology, 5:400-404.

Date of initial receipt: 22 February 1993

Date of acceptance: 28 February 1994

Ms 138SR-150 


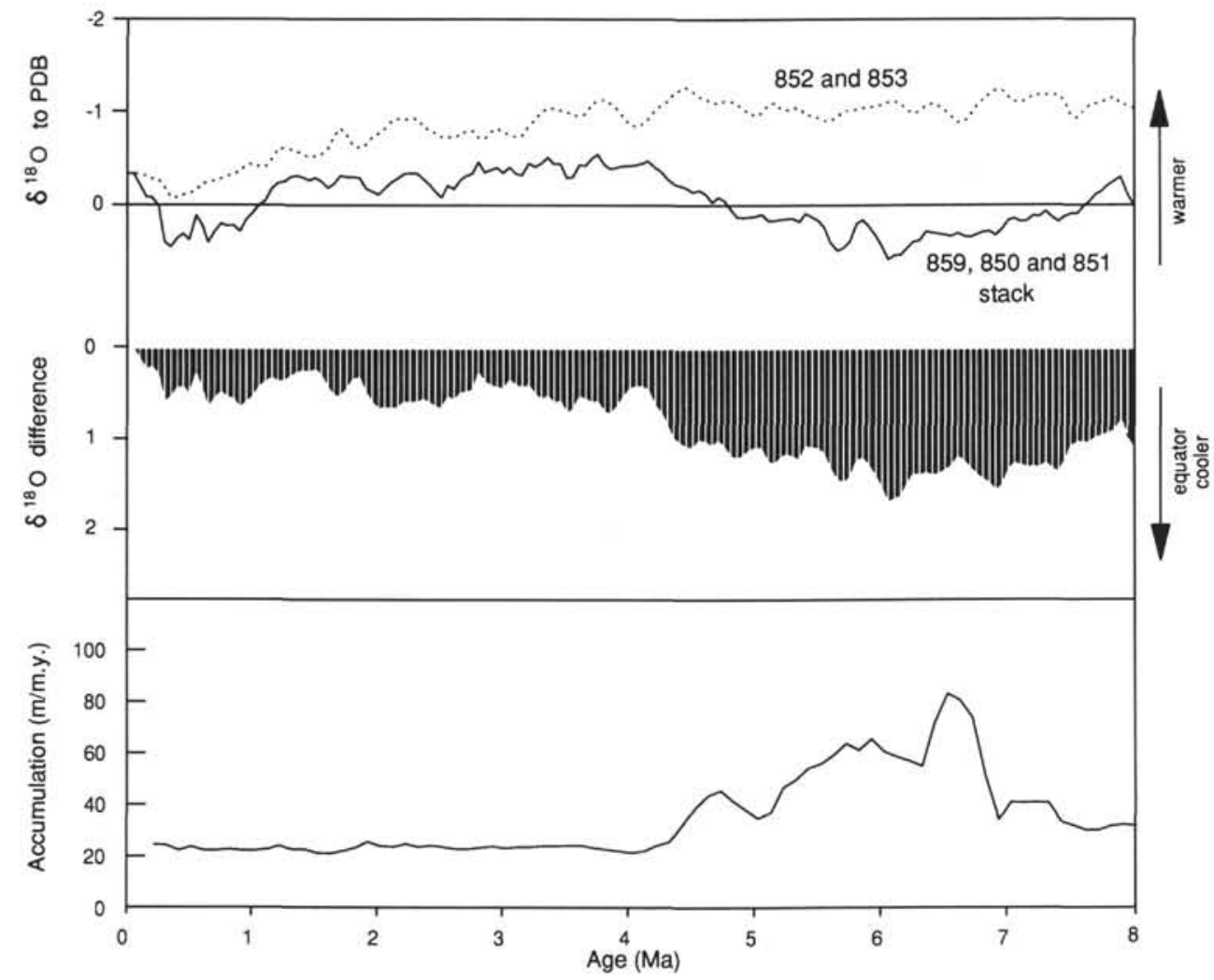

Figure 6. Smoothed $\delta^{18} \mathrm{O}$ record (above) of off-equator Sites 852 and 853 , compared with that of near-equatorial Sites 849,850 , and $851 . \delta^{18} \mathrm{O}$ gradient (middle, shaded) between about $5^{\circ} \mathrm{N}$ and the equator (the difference between the above records). Average sediment accumulation rates (below) in Sites 849, 850, and 851 . 